\section{(6) OPEN ACCESS}

\title{
Relationship between exposure to tumour necrosis factor inhibitor therapy and incidence and severity of myocardial infarction in patients with rheumatoid arthritis
}

\author{
Audrey S L Low, ${ }^{1}$ Deborah P M Symmons, ${ }^{1,2}$ Mark Lunt, ${ }^{1}$ Louise K Mercer, ${ }^{1}$ Chris \\ P Gale, ${ }^{3,4}$ Kath D Watson, ${ }^{1}$ William G Dixon, ${ }^{1}$ Kimme L Hyrich, ${ }^{1}$ on behalf of the \\ British Society for Rheumatology Biologics Register for Rheumatoid Arthritis (BSRBR- \\ RA) and the BSRBR Control Centre Consortium
}

\begin{abstract}
Handling editor Tore K Kvien
- Additional material is published online only. To view please visit the journal online (http://dx.doi.org/10.1136/ annrheumdis-2016-209784)

For numbered affiliations see end of article.

\section{Correspondence to} Professor Kimme Hyrich, Arthritis Research UK Centre for Epidemiology, Centre for Musculoskeletal Research, Institute of Inflammation and Repair, The University of Manchester, Manchester Academic Health Science Centre, Stopford Building, Oxford Road, Manchester M13 9PT, UK; Kimme.Hyrich@ manchester.ac.uk
\end{abstract}

Received 25 April 2016 Revised 27 July 2016 Accepted 12 August 2016 Published Online First 4 January 2017

\section{CrossMark}

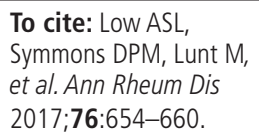

\section{ABSTRACT}

Objectives Patients with rheumatoid arthritis (RA) are at increased risk of myocardial infarction (MI) compared with subjects without RA, with the increased risk driven potentially by inflammation. Tumour necrosis factor inhibitors (TNFi) may modulate the risk and severity of MI. We compared the risk and severity of MI in patients treated with TNFi with that in those receiving synthetic disease-modifying antirheumatic drugs (sDMARDs). Methods This analysis included patients with RA recruited from 2001 to 2009 to the British Society for Rheumatology Biologics Register for Rheumatoid Arthritis starting TNFi (etanercept/infliximab/adalimumab) and a biologic-naïve comparator cohort receiving sDMARD. All patients were followed via physician and patient questionnaires and national death register linkage. Additionally, all patients were linked to the Myocardial Ischaemia National Audit Project, a national registry of hospitalisations for MI. Patients were censored at first verified $\mathrm{MI}$, death, 90 days following TNFi discontinuation, last physician follow-up or 20 April 2010, whichever came first. The risk of first MI was compared between cohorts using COX regression, adjusted with propensity score deciles (PD). MI phenotype and severity were compared using descriptive statistics. 6-month mortality post MI was compared using logistic regression.

Results 252 verified first Mls were analysed: 58 in 3058 patients receiving sDMARD and 194 in 11200 patients receiving TNFi (median follow-up per person 3.5 years and 5.3 years, respectively). The PD-adjusted $\mathrm{HR}$ of $\mathrm{MI}$ in TNFi referent to sDMARD was $0.61(95 \% \mathrm{Cl}$ 0.41 to 0.89 ). No statistically significant differences in $\mathrm{Ml}$ severity or mortality were observed between treatment groups.

Conclusions Patients with RA receiving TNFi had a decreased risk of MI compared with patients with RA receiving SDMARD therapy over the medium term. This might be attributed to a direct action of TNFi on the atherosclerotic process or better overall disease control.

\section{INTRODUCTION}

In meta-analyses, patients with rheumatoid arthritis (RA) have a $60 \%$ increased risk of myocardial infarction (MI) and a 70\% increased risk in mortality from
MI compared with the general population. ${ }^{12}$ As the development of atherosclerosis in the general population is viewed as an inflammatory process, it is possible that the chronic inflammation associated with RA may accelerate this. Traditional cardiovascular (CV) risk factors do not fully explain the increased risk of MI associated with RA. ${ }^{3-5}$ Drugs inhibiting tumour necrosis factor $\alpha$ (TNFi) have been shown to reduce joint inflammation and associated inflammatory markers; thus, they may also influence the future risk of MI.

The association between TNFi exposure and MI risk has been investigated previously in patients with RA. Some studies found a reduced risk, but others a similar risk compared with treatment with synthetic disease-modifying antirheumatic drugs (sDMARDs). ${ }^{6-15}$ Most of these studies only followed patients for 1-2 years. TNFi may influence the incidence of $\mathrm{MI}$ in the short term by stabilising plaque. However, any effect on plaque formation is likely to take much longer. Therefore, the full influence of TNFi on future MI risk may take many years to become apparent. ${ }^{16}$ Also, as $\mathrm{MI}$ is a relatively uncommon event, large sample sizes are required to assess this risk.

As well as influencing the occurrence of $\mathrm{MI}$, tumour necrosis factor $\alpha(\mathrm{TNF} \alpha)$ may affect the outcome after a CV event. TNF $\alpha$ appears to limit infarct size by preventing or delaying apoptosis of cardiac myocytes and may have a homeostatic role in limiting the amount and duration of damage after an ischaemic insult. ${ }^{17}$ Conversely, neutralising $\mathrm{TNF} \alpha$ with antibodies has been shown to reduce infarct size in murine models. ${ }^{18}$ The outcome of MI in patients with RA receiving TNFi therapy has not previously been studied.

We aimed to compare (1) the incidence of MI over the medium term, (2) the severity of MI and (3) the mortality post MI between patients with RA treated with TNFi therapy and those treated with sDMARD therapy.

\section{METHODS}

\section{Study design and setting}

The British Society for Rheumatology Biologics Register for Rheumatoid Arthritis (BSRBR-RA) is a 
UK-wide prospective observational study, established in 2001 to monitor the long-term safety of TNFi and other biological therapies. ${ }^{19}$ UK guidelines restrict the prescription of TNFi in RA to patients with (i) sustained active disease (28-joint disease activity score (DAS28) $>5.1$ on at least two occasions a month apart) and (ii) who have failed to respond to therapeutic doses of $\geq 2$ sDMARDs (including methotrexate, unless contraindicated) given for $\geq 6$ months. ${ }^{20}$ The TNFi-treated patients included in this analysis were recruited between 2001 and 2005 (etanercept), 2001 and 2008 (infliximab) and 2004 and 2008 (adalimumab). Recruitment to each TNFi drug continued from when that TNFi was licensed in UK until the target of 4000 patients per TNFi was reached. We recruited a comparator cohort of biologic-naïve patients with active disease (guide DAS28>4.2) receiving sDMARD therapies only, between 2002 and 2009. If patients in the comparator cohort were switched to TNFi therapy, they were given the option to re-consent to recruitment to the TNFi cohort (if recruitment to that TNFi cohort was still open); otherwise follow-up was discontinued. Patients could not switch from the TNFi cohort to the sDMARD cohort.

Both cohorts were followed identically via physician and patient questionnaires. Questionnaires were sent to the rheumatology team every 6 months for the first 3 years of follow-up and annually thereafter, regardless of changes to therapy, requesting information on disease details, medication and comorbidities, as well as the occurrence of adverse events. At baseline, physicians were asked if the patient had a history of MI or angina. Patients provided information on hospitalisations via questionnaires every 6 months for the first 3 years of follow-up. For all reports of MI, additional clinical data (discharge summaries, ECG, cardiac enzymes, pathology reports) were requested to aid event verification and classification. All patients were flagged with the Health and Social Care Information Centre for reporting of deaths. Causes of death (COD) were coded from the death certificate using the International Classification of Diseases 10.

In addition, the BSRBR-RA dataset was linked to the Myocardial Ischaemia National Audit Project (MINAP). Established in 1999, MINAP audits the care and outcome of all patients admitted to an acute National Health Service (NHS) hospital in England or Wales with a suspected MI against national standards. ${ }^{21}$ MINAP collects patient-level information including patient demographics, dates of admission, MI phenotype, baseline risk, comorbidities, investigations, details of reperfusion therapy, in-hospital drug treatment, clinical complications and all-cause mortality.

The BSRBR-RA and MINAP datasets were linked using deterministic matching based on surname, forename, gender, unique NHS number, date of birth and postcode. The matching strategy used a maximum of four linkage variables in any one combination. MINAP events which matched to a BSRBR-RA patient were returned to the BSRBR-RA for analysis.

\section{Participants}

This analysis included all patients with a physician diagnosis of RA starting etanercept, infliximab or adalimumab as their first biologic within 6 months of registration with BSRBR-RA, or registered into the SDMARD cohort (figure 1). The analysis was limited to patients with at least moderate disease activity at the start of therapy (DAS28 $\geq 3.2$ ) and with no past history of MI or angina. All patients had to have at least one returned rheumatology team follow-up questionnaire to confirm treatment start.

Written consent was obtained from all patients according to the Declaration of Helsinki. Approval for the BSRBR-RA was given by

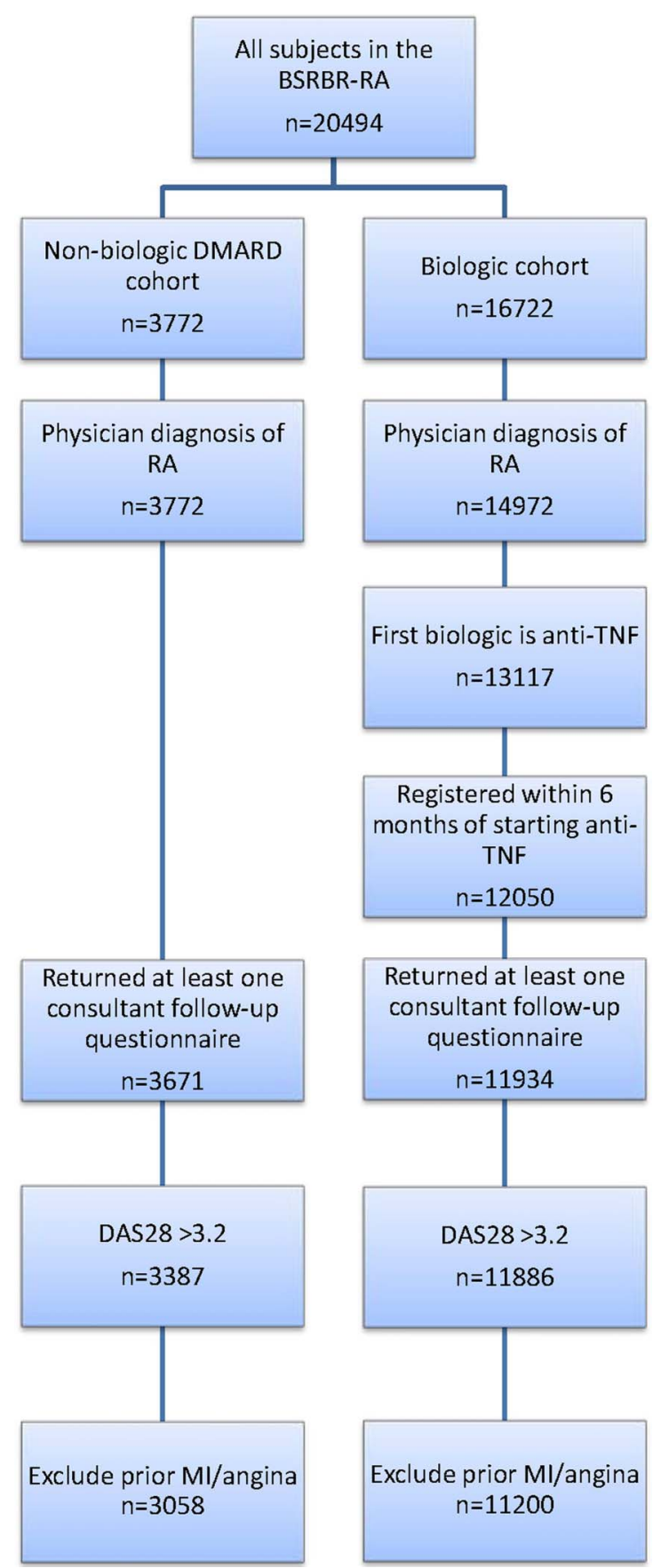

Figure 1 Patient selection for analysis. BSRBR-RA, British Society for Rheumatology Biologics Register for Rheumatoid Arthritis; DAS28, disease activity in 28 joints; DMARD, disease-modifying antirheumatic drug; MI, myocardial infarction; RA, rheumatoid arthritis; TNF, tumour necrosis factor.

the North West Multicentre Research Ethics Committee (reference no: 00/8/53). The National Institute for Cardiovascular Outcomes Research (NICOR) which includes MINAP (Ref: NIGB: ECC 1-06 (d)/2011) has support under Section 251 of the NHS Act 2006 to use patient information for medical research without requiring additional consent. The data linkage in this analysis was approved by the MINAP Academic Group.

\section{Verification of MI}

All potential MIs reported to BSRBR-RA, MINAP or both were verified using the American Heart Association/European Society 
of Cardiology (AHA/ESC) criteria for MI. ${ }^{22}$ If a reported event could not be verified against the AHA/ESC criteria for MI, but the patient received thrombolysis or primary angioplasty or died with MI recorded as the underlying COD, this was also considered to be a verified MI.

\section{Drug exposure models and statistical methods}

The primary event was the first verified MI for each patient. Crude incidence rates of first MI with 95\% CIs were calculated using a Poisson distribution. For the TNFi cohort, follow-up started on the first day of treatment. For the sDMARD cohort, follow-up started on the date of registration. MIs were attributed to TNFi therapy if they occurred on drug or within 90 days of drug discontinuation. Follow-up was censored at first verified MI, death, date of last physician follow-up or 20 April 2010, whichever came first. The risk of first MI was compared between TNFi-treated and sDMARD-treated patients using a COX proportional hazards model, adjusted for deciles of propensity score (PD). This was presented using HRs with 95\% CI. Sensitivity analyses included (1) analysing the data using an everexposed drug model (ie, all exposure time following first dose of TNFi) and (2) trimming of the PD at 5\%.

Baseline confounders were specified a priori and entered into a logistic regression model to generate a PD, reflecting the likelihood of receiving TNFi depending on covariates. These covariates were age, gender, DAS28, disease duration, health assessment questionnaire score, whether the patient had used $\geq 4$ sDMARDs prior to study registration, whether the patient was recruited to the register before 30 June 2004 (the approximate midpoint of study recruitment, chosen to account for temporal changes in baseline disease severity over time), ${ }^{23}$ hypertension, diabetes, chronic lung disease, smoking (ever/ never), antiplatelet therapy, non-steroidal anti-inflammatory drugs (NSAID)/cyclooxygenase inhibitor (COX)-2 inhibitor use, glucocorticoid use and statin use. Missing data were imputed by multiple imputation by chained equations (MICE). ${ }^{24}$ The imputation model included whether the patient experienced an MI, logarithm of the time to MI and the other covariates as described $^{25}$ (see online supplementary web appendix).

Severity of the MI was defined according to (1) MI phenotype: ST elevation versus non-ST elevation (STEMI vs NSTEMI), (2) presence or absence of cardiac arrest during hospital admission, (3) peak creatine kinase (CK), peak troponin I, peak troponin $\mathrm{T}$ and (4) length of hospital stay. These parameters were compared between cohorts using the $\chi^{2}$ test for categorical variables and the Wilcoxon rank-sum test for continuous variables. This analysis was limited to those MIs captured in the MINAP registry. For this analysis, patients were divided into three groups based on treatment at the time of their MI: Group 1 (sDMARD) (referent), Group 2 (receiving TNFi therapy at the time of or within 90 days prior to the MI) and Group 3 (exposed to TNFi but outside the 90-day lag window prior to the MI).

Mortality post MI was defined as any death that occurred within 6 months following the initial MI. The results are presented for (1) all MIs irrespective of reporting source and (2) for those MIs with additional data from MINAP. Using logistic regression, the risk of death within 6 months post MI was compared between the three groups, with the sDMARD group as the referent. For all MIs, mortality was adjusted for age and gender. For those MIs with additional data from MINAP, the analysis was adjusted for age, gender and the Modified Global Registry of Acute Coronary Events (MG) score: a composite score including age, heart rate, systolic blood pressure, creatinine, cardiac arrest at admission, ST segment deviation, elevated cardiac enzymes and loop diuretic use at admission. ${ }^{26} 27$ Higher scores indicate a higher probability of death (range: 0 274). Missing data for components of MG were replaced using multiple imputation. ${ }^{28}$ The risk of death was estimated using logistic regression. ORs with 95\% CI were presented. All analyses were performed using Stata V.13 (StataCorp, College Station, Texas, USA).

\section{RESULTS}

\section{Baseline characteristics}

A total of 14258 patients (sDMARD: 3058, TNFi: 11200 ) were analysed (table 1 and figure 1). At baseline, the TNFi cohort was younger, comprised of proportionally more females, had longer disease duration and higher disease activity and functional disability compared with the sDMARD cohort. Patients in the TNFi cohort were also more likely to receive glucocorticoids and NSAID/COX-2 inhibitors, but were less likely to be on antiplatelet drugs and statins. Patients in the TNFi cohort also had a lower frequency of smoking, hypertension and diabetes compared with the sDMARD cohort. Median duration of exposure to TNFi was 4.1 years (IQR 2.0, 5.8).

\section{Risk of MI in TNFi-treated patients compared with sDMARD-treated patients}

There were 58 verified first MIs during a median of 3.5 years follow-up in the sDMARD cohort (total follow-up 10337 . person-years (pyrs)) and 194 MIs during a median of 5.3 years follow-up in the TNFi cohort (total follow-up 55636 pyrs) (table 2). The crude incidence rate of first MIs per 10000 pyrs was 56 (95\% CI 46 to 73$)$ in the sDMARD cohort and 35 (95\% CI 30 to 40 ) in the TNFi cohort. The median time to first MI was 1.56 years (IQR $0.89,3.43$ ) in the sDMARD cohort and 2.43 years (IQR $1.41,3.96$ ) in the TNFi cohort.

\begin{tabular}{|c|c|c|}
\hline & $\begin{array}{l}\text { SDMARD; } \\
\mathrm{n}=3058\end{array}$ & $\begin{array}{l}\text { TNFi; } \\
n=11200\end{array}$ \\
\hline Mean age, years (SD) & $59.5(12.5)$ & $55.6(12.3)$ \\
\hline Female, \% & 75 & 78 \\
\hline Median disease duration, years (IQR) & $6(1,15)$ & $11(6,19)$ \\
\hline Mean DAS28 (SD) & $5.3(1.1)$ & $6.6(1.0)$ \\
\hline Mean HAQ score (SD) & $1.5(0.7)$ & $2.0(0.6)$ \\
\hline $\begin{array}{l}\text { Proportion of patients who received } \geq 4 \\
\text { sDMARDs prior to study registration, } \%\end{array}$ & 21 & 53 \\
\hline $\begin{array}{l}\text { Proportion of patients who received } \\
\text { methotrexate prior to study registration, \% }\end{array}$ & 82 & 97 \\
\hline Recruited before 30 June $2004, \%$ & 19 & 51 \\
\hline Hypertension, \% & 30 & 28 \\
\hline Diabetes, \% & 6 & 5 \\
\hline Chronic lung disease, $\%$ & 19 & 13 \\
\hline Current/previous smoker, $\%$ & 62 & 59 \\
\hline Glucocorticoid, \% & 22 & 44 \\
\hline NSAID/COX-2 inhibitor therapy, \% & 56 & 63 \\
\hline Antiplatelet therapy, \% & 7 & 5 \\
\hline Statin therapy, \% & 9 & 5 \\
\hline \multicolumn{3}{|c|}{$\begin{array}{l}\text { COX, cyclooxygenase inhibitor; DAS28, disease activity in } 28 \text { joints; HAQ score, health } \\
\text { assessment questionnaire score; NSAID, non-steroidal anti-inflammatory drugs; } \\
\text { sDMARD, synthetic disease-modifying antirheumatic drug; TNFi, tumour necrosis factor } \\
\alpha \text { inhibitor. }\end{array}$} \\
\hline
\end{tabular}




\begin{tabular}{|c|c|c|}
\hline & $\begin{array}{l}\text { SDMARD; } \\
\mathrm{n}=3058\end{array}$ & TNFi; $n=11200$ \\
\hline $\begin{array}{l}\text { Median duration of follow-up per patient, } \\
\text { years (IQR) }\end{array}$ & $3.5(1.8,4.9)$ & $5.3(3.6,6.4)$ \\
\hline Total person-years of exposure, pyrs & 10337 & 55636 \\
\hline \multicolumn{3}{|l|}{ Primary drug exposure model: on-TNFi+90 days } \\
\hline Number of verified first Mls & 58 & 194 \\
\hline $\begin{array}{l}\text { Crude incidence rate of verified first MI } \\
\text { per } 10000 \text { pyrs }(95 \% \mathrm{Cl})\end{array}$ & 56 (43 to 73 ) & 35 (30 to 40$)$ \\
\hline Unadjusted HR $(95 \% \mathrm{Cl})$ & Referent & 0.78 (0.58 to 1.05$)$ \\
\hline $\begin{array}{l}\text { HR adjusted for age and } \\
\text { gender }(95 \% \mathrm{Cl})\end{array}$ & & 1.19 (0.89 to 1.59$)$ \\
\hline HR after adjusting for $\mathrm{PD}^{*}(95 \% \mathrm{Cl})$ & & 0.61 (0.41 to 0.89$)$ \\
\hline \multicolumn{3}{|l|}{ Sensitivity analyses } \\
\hline $\begin{array}{l}\text { In subjects ever exposed to TNFi; } \\
\text { PD-adjusted HR ( } 95 \% \mathrm{CI})\end{array}$ & & 0.67 (0.46 to 0.96$)$ \\
\hline $\begin{array}{l}\text { Trimming the PD at 5\%; PD-adjusted } \\
\text { HR }(95 \% \mathrm{Cl})\end{array}$ & & $0.56(0.34$ to 0.93$)$ \\
\hline
\end{tabular}

*Deciles of propensity score (PD). The PD included age, gender, DAS28, disease duration, health assessment questionnaire score, whether the patients used four or more sDMARDs prior to study registration (yes/no), whether the patients were recruited to the register before or after 30 June 2004, hypertension, diabetes, chronic lung disease, smoking (ever/never), antiplatelet therapy, NSAID/COX-2 inhibitor use, glucocorticoid use and statin use.

COX, cyclooxygenase inhibitor; DAS28, disease activity in 28 joints; Ml, myocardial infarction; NSAID, non-steroidal anti-inflammatory drugs; SDMARD, synthetic disease-modifying antirheumatic drug; TNFi, tumour necrosis factor $\alpha$ inhibitor.

Compared with the sDMARD cohort, the unadjusted HR of MI in the TNFi cohort was 0.78 (95\% CI 0.58 to 1.05). After adjustment using $\mathrm{PD}$, the risk of $\mathrm{MI}$ in the TNFi cohort was 0.61 (95\% CI 0.41 to 0.89 ), indicating a $39 \%$ decreased risk of MI, compared with the sDMARD cohort. There were 276 verified MIs in the group who were ever exposed to TNF (ie, when all person-time following first dose of TNFi was included). Adjustment using PD showed a result similar to the primary model: HR 0.67 (95\% CI 0.46 to 0.96) (table 2). Similar results were also found when propensity scores were trimmed at 5\% (table 2).

\section{Severity of MI}

Of the 334 verified first MIs (sDMARD: 58, ever exposed to TNFi: 276), 136 MIs were captured by BSRBR-RA only, 78 were captured by MINAP only and 120 were captured in both datasets. There were no significant differences (data not shown) in the age, gender or the proportion of patients receiving TNFi therapy in those MIs with MINAP data and those without.

Of the 198 MI patients with MINAP data (59\%), 35 were biologic-naïve (Group 1), 108 were receiving TNFi therapy at the time of or within 90 days prior to the MI (Group 2) and 55 had prior exposure to TNFi (Group 3) (table 3). Overall, there were no statistically significant differences in markers of severity between the three groups, although there was a trend towards higher peak CK in patients on TNFi at the time of MI (table 3).

\section{Post-MI mortality}

Of the 334 patients with first MIs, 77 (23\%) died within 6 months of their MI: $12(21 \%)$ in Group 1, 25 (13\%) in Group 2 and 40 (48\%) in Group 3 (exposed, but had stopped TNFi $>90$ days before their MI) (table 4). Compared with the sDMARD-treated group (Group 1), the age and gender adjusted OR of death in Group 2 was 0.68 (95\% CI 0.31 to 1.47) and that in Group 3 was 3.07 (95\% CI 1.42 to 6.62). The median time between stopping the TNFi and MI occurrence was 1.3 years (IQR $0.5,2.5)$. Similar trends in mortality risk were observed in the subset of MIs captured by MINAP, but there were very few events, and precision was low (table 4). The median MG scores were similar between the three groups (108 vs 100 vs 112 , respectively). The MG score was a significant univariate predictor of 6 -month mortality: OR 1.05 (95\% CI 1.02 to 1.07 ) within these subjects.

\section{DISCUSSION}

Previous reports of the association between TNFi therapy and the risk of MI have only followed patients up to 1-2 years and have not had consistent findings. We examined the association between TNFi therapy and the risk of MI over the medium term (median follow-up, 5 years) and used propensity scores to balance differences across a wide range of measured covariates. A 39\% reduction in the risk of MI was observed in patients treated with TNFi compared with those on sDMARD therapy. We also report, for the first time, the relationship between severity of and mortality post MI among patients who have received TNFi therapy.

There is a signal that duration of TNFi exposure is related to a reduction in cardiovascular disease (CVD) risk in patients with RA. Bili et $a l^{13}$ found that use of TNFi for more than the median of 16 months was associated with lower risk of CV events: relative risk 0.31 (95\% CI 0.15 to 0.65$)$. In a Swedish study, 2 years follow-up on TNFi was associated with a $32 \%$

\begin{tabular}{|c|c|c|c|c|}
\hline $\begin{array}{l}\text { Number of verified first Mls with } \\
\text { additional MINAP data }\end{array}$ & $\begin{array}{l}\text { Group } 1 \text { (sDMARD), } \\
\mathrm{n}=35\end{array}$ & $\begin{array}{l}\text { Group } 2 \text { (on TNFi at the time of or within } \\
90 \text { days prior to MI), } n=108\end{array}$ & $\begin{array}{l}\text { Group } 3 \text { (exposure to TNFi more than } \\
90 \text { days prior to MI), } n=55\end{array}$ & $\mathrm{p}$ Value \\
\hline $\begin{array}{l}\text { Proportion of patients with STEMI, } \mathrm{n} \\
(\%)\end{array}$ & $16(46)$ & $53(49)$ & $27(49)$ & 0.32 \\
\hline Cardiac arrest, $\mathrm{n}(\%)$ & $3(9)$ & $5(5)$ & $5(9)$ & 0.48 \\
\hline Median peak CK, IU/L (IQR) & $290(172,1598)$ & $691(150,1293)$ & $286(125,660)$ & 0.19 \\
\hline Median peak troponin $\mathrm{I}, \mu \mathrm{g} / \mathrm{L}(\mathrm{IQR})$ & $5.0(1.3,7.2)$ & $7.4(1.1,22.8)$ & $7.6(1.5,29.0)$ & 0.46 \\
\hline Median peak troponin $\mathrm{T}, \mu \mathrm{g} / \mathrm{L}(\mathrm{IQR})$ & $0.7(0.3,2.3)$ & $0.9(0.2,2.3)$ & $0.8(0.2,2.1)$ & 0.95 \\
\hline $\begin{array}{l}\text { Median length of hospital stay, days } \\
\text { (IQR) }\end{array}$ & $6(5,9)$ & $6(4,8)$ & $6(4,11)$ & 0.46 \\
\hline
\end{tabular}

CK, creatine kinase; MI, myocardial infarction; MINAP, Myocardial Ischaemia National Audit Project; SDMARD, synthetic disease modifying anti-rheumatic drug; STEMI, ST-elevation myocardial infarction; TNFi, tumour necrosis factor $\alpha$ inhibitor. 
Table 4 Mortality within the 6-months following MI

\begin{tabular}{|c|c|c|c|}
\hline & Group 1 (sDMARD) & $\begin{array}{l}\text { Group } 2 \text { (on TNFi+90 days } \\
\text { lag at time of MI) }\end{array}$ & $\begin{array}{l}\text { Group } 3 \text { (exposure to TNFi more } \\
\text { than } 90 \text { days prior to } \mathrm{MI} \text { ) }\end{array}$ \\
\hline Total number of verified first Mls identified from BSRBR-RA and/or MINAP & 58 & 194 & 82 \\
\hline Deaths within 6 months, $\mathrm{n}(\%)$ & $12(21)$ & $25(13)$ & $40(48)$ \\
\hline Unadjusted OR $(95 \% \mathrm{Cl})$ & Referent & 0.61 (0.28 to 1.31$)$ & 2.84 (1.33 to 6.04$)$ \\
\hline OR adjusted for age and gender $(95 \% \mathrm{Cl})$ & & $0.68(0.31$ to 1.47$)$ & 3.07 (1.42 to 6.62$)$ \\
\hline Number of verified first Mls with MINAP data (\% total verified MIs) & $35(60)$ & $108(56)$ & $55(67)$ \\
\hline Deaths within 6 months, $\mathrm{n}(\%)$ & $2(6)$ & $3(3)$ & $11(20)$ \\
\hline Median MG score (IQR) & $108(81$ to 131$)$ & $100(84$ to 120$)$ & 112 (93 to 129) \\
\hline Unadjusted OR $(95 \% \mathrm{Cl})$ & Referent & 0.47 (0.08 to 2.94$)$ & $4.13(0.86$ to 19.89$)$ \\
\hline OR adjusted for age and gender $(95 \% \mathrm{Cl})$ & & $0.51(0.08$ to 3.21$)$ & 4.07 (0.82 to 20.07$)$ \\
\hline OR adjusted for $\mathrm{MG}$ score $(95 \% \mathrm{Cl})$ & & $0.47(0.06$ to 3.45$)$ & $5.40(0.93$ to 31.18$)$ \\
\hline
\end{tabular}

BSRBR-RA, British Society for Rheumatology Biologics Register for Rheumatoid Arthritis; MG score, modified Global Registry of Acute Coronary Events score; MI, myocardial infarction; MINAP, Myocardial Ischaemia National Audit Project; SDMARD, synthetic disease modifying anti-rheumatic drug; TNFi, tumour necrosis factor $\alpha$ inhibitor.

reduction in the risk of acute coronary syndrome: HR 0.78 (95\% CI 0.61 to 1.01$){ }^{29}$ There is a biologically plausible explanation for our findings as TNF $\alpha$ plays a key role in the pathogenesis of atherosclerosis. ${ }^{30}$ Inflammation is central in all stages of atherosclerosis, including endothelial function, plaque stabilisation and postinfarct remodelling, and thus inhibition of $\mathrm{TNF} \alpha$ may influence accumulation and progression of plaque leading to fewer MIs. TNFi may also affect CVD risk via changes in lipid profile, insulin resistance and diabetes risk. ${ }^{31} 32$

It is possible that our study findings are attributable to suppression of inflammation and disease control in general rather than a TNF $\alpha$-specific effect. Solomon et $a l^{33}$ observed that disease activity control was associated with fewer CV events. Therefore, the current treat-to-target strategy to lower disease activity in RA may improve pain and function, and also reduce $\mathrm{CV}$ risk either through using sDMARDs or biologics or a combination. In our study, disease activity in the comparator cohort may have been suppressed by SDMARD therapy, but to a lesser extent, than in the TNFi cohort, thereby 'maintaining' the already increased background MI risk.

Blockade of TNF $\alpha$ may modify the incidence of MI and influence the severity and mortality post MI via postinfarct remodelling. ${ }^{17} 18$ This relationship has not previously been explored because the relevant data are generally not collected within drug registries. For this study, we linked with a national MI database to gain additional data on the MIs. Due to differences in study design and geographical setting between MINAP and BSRBR-RA, the overlap of events was not $100 \%$. However, there were no systematic differences between those with MINAP data and those without. Overall, there were no differences in MI severity between treatment groups using indirect measures (MI phenotype, in-hospital cardiac arrest, troponin levels and length of hospital stay). We observed a trend towards higher peak CK levels in patients on TNFi at the time of MI. However, data on the precise time from MI symptom onset to measurement of cardiac enzymes in relation to reperfusion treatment were not recorded in MINAP. These parameters may influence our results; thus, this finding should be interpreted with caution.

Mortality post MI in the general population is associated with a number of factors (eg, age, gender, severity of MI, comorbidities). We were able to use data from MINAP to calculate the modified GRACE score and include this in the regression model. However, small numbers of events precluded robust conclusions. We observed a difference in the direction of relative risk between the group of patients receiving TNFi at the time of MI and those with prior exposure to TNFi $(50 \%$ reduction vs fivefold increase, respectively). In the latter group, the median time between stopping the TNFi and MI was 1.3 years. Most patients in the latter group had discontinued their TNFi following an adverse event (MI was not the adverse event), which may imply higher levels of comorbidity in these patients.

This study has several strengths. The prospective design of the BSRBR-RA, detailed data collection and the size of the study population meant that it was possible to adjust for a large number of potential confounders compared with previous publications. Despite the large range of covariates, we were unable to adjust for unmeasured confounders such as cumulative steroid dose. Confounding by indication is an issue with observational studies. If patients with severe active RA are at increased risk of CVD and are also more likely to receive TNFi, one would have expected an increased MI risk in the TNFi group, but instead a reduced risk was observed. Employment of propensity scores to balance confounders is an emerging technique in the field of pharmacoepidemiology. In this analysis, the use of PD to adjust for known confounders was associated with low levels of expected bias ( $<5 \%$, see online supplementary web appendix). Linkage with MINAP enabled analysis of MI severity, including adjustment by the modified GRACE score (a risk prediction score for MI-related death). This study, which has looked at the effects of TNFi when added to sDMARD therapy, cannot be used to compare the risk of MI between incident TNFi use and incident sDMARD use.

In conclusion, treatment with TNFi therapy for RA was associated with a reduced risk of MI over the medium term compared with sDMARD therapy. This might be attributed to a direct action of TNFi on the atherosclerotic process or better overall disease control or both. Severity of MI and mortality post MI were not associated with TNFi therapy in our dataset, but warrants further exploration in collaborative analyses across other biologic registers.

\section{Author affiliations \\ ${ }^{1}$ Arthritis Research UK Centre for Epidemiology, Centre for Musculoskeletal Research, Institute of Inflammation and Repair, The University of Manchester, Manchester, UK ${ }^{2}$ NIHR Manchester Musculoskeletal Biomedical Research Unit, Central Manchester NHS Foundation Trust, Manchester Academic Health Science Centre, Manchester, UK ${ }^{3}$ Division of Epidemiology and Biostatistics, Leeds Institute of Cardiovascular and Metabolic Medicine, University of Leeds, Leeds, UK \\ ${ }^{4}$ York Teaching Hospital NHS Foundation Trust, York, UK}

Twitter Follow the Centre for Musculoskeletal Research at @UoMMskResearch 
Acknowledgements The authors acknowledge the enthusiastic collaboration of all consultant rheumatologists and their specialist nurses in the UK in providing the data. The authors would like to gratefully acknowledge the support of the National Institute for Health Research, through the comprehensive local research networks at participating centres. In addition, the authors acknowledge support from the British Society for Rheumatology (BSR) Executive, the members of the BSR Registers and Research Committee and the BSRBR-RA Project Team in London for their active role in enabling the register to undertake its tasks. The authors also acknowledge the seminal role of the BSR Clinical Affairs Committee for establishing national biological guidelines and recommendations for such a register. We acknowledge gratefully the support of the hospitals and staff across England and Wales who contributed to the collection of MINAP data, the MINAP academic group (Chair, Professor A Timmis) and the NICOR. The authors also acknowledge the Arthritis Research UK Centre for Epidemiology (Grant No. 20380) for infrastructure and technical support in data collection and statistical analysis.

Collaborators BSRBR Control Centre Consortium: The BSRBR Control Centre Consortium consists of the following institutions (all in the UK): Antrim Area Hospital, Antrim (Dr Nicola Maiden); Cannock Chase Hospital, Cannock Chase (Dr Tom Price); Christchurch Hospital, Christchurch (Dr Neil Hopkinson); Royal Derby Hospital, Derby (Dr Sheila O'Reilly), Dewsbury and District Hospital, Dewsbury (Dr Lesley Hordon); Freeman Hospital, Newcastle upon Tyne (Dr lan Griffiths); Gartnavel General Hospital, Glasgow (Dr Duncan Porter); Glasgow Royal Infirmary, Glasgow (Professor Hilary Capell); Haywood Hospital, Stoke-on-Trent (Dr Andy Hassell); Hope Hospital, Salford (Dr Romela Benitha); King's College Hospital, London (Dr Ernest Choy); Kings Mill Centre, Sutton-in Ashfield (Dr David Walsh); Leeds General Infirmary, Leeds (Professor Paul Emery); Macclesfield District General Hospital, Macclesfield (Dr Susan Knight); Manchester Royal Infirmary, Manchester (Professor Ian Bruce); Musgrave Park Hospital, Belfast (Dr Allister Taggart); Norfolk and Norwich University Hospital, Norwich (Professor David Scott); Poole General Hospital, Poole (Dr Paul Thompson); Queen Alexandra Hospital, Portsmouth (Dr Fiona McCrae); Royal Glamorgan Hospital, Glamorgan (Dr Rhian Goodfellow); Russells Hall Hospital, Dudley (Professor George Kitas); Selly Oak Hospital, Selly Oak (Dr Ronald Jubb); St Helens Hospital, St Helens (Dr Rikki Abernethy); Weston General Hospital, Weston-super-Mare (Dr Shane Clarke/Dr Sandra Green); Withington Hospital, Manchester (Dr Paul Sanders); Withybush General Hospital, Haverfordwest (Dr Amanda Coulson); North Manchester General Hospital (Dr Bev Harrison); Royal Lancaster Infirmary (Dr Marwan Bukhari) and The Royal Oldham Hospital (Dr Peter Klimiuk).

Funding This work was supported by the BSR. The BSR commissioned the BSRBR-RA as a UK-wide national project to investigate the safety of biological agents in routine medical practice. BSR receives restricted income from UK pharmaceutical companies, presently Abbvie, Celltrion, Hospira, Pfizer, UCB and Roche, and in the past Swedish Orphan Biovitrum and Merck. This income finances a wholly separate contract between the BSR and the University of Manchester. The pharmaceutical company funders had no role in the design and conduct of the study; collection, management, analysis and interpretation of the data; preparation, review or approval of the manuscript; and decision to submit the manuscript for publication. Members of the University of Manchester team, BSR trustees, committee members and staff complete an annual declaration in relation to conflicts of interest. All relevant information regarding serious adverse events outlined in the manuscript have been reported to the appropriate companies as per the contractual agreements/ standard operating procedures. The British MINAP is commissioned by the Health Quality Improvement Partnership as part of the National Clinical Audit and Patient Outcomes Programme. CPG is funded by the National Institute for Health Research (NIHR-CTF-2014-03-03) as Associate Professor and Honorary Consultant Cardiologist. WGD was supported by an MRC Clinician Scientist Fellowship (G0902272). DPMS and KH are the principal investigators of the BSRBR-RA and had full access to all the data in the study and take responsibility for the integrity of the data and the accuracy of the data analysis.

Competing interests $\mathrm{KH}$ has received honoraria from Pfizer and Abbvie.

Ethics approval UK North West Multicentre Research Ethics Committee (reference no: 00/8/53).

Provenance and peer review Not commissioned; externally peer reviewed.

Open Access This is an Open Access article distributed in accordance with the terms of the Creative Commons Attribution (CC BY 4.0) license, which permits others to distribute, remix, adapt and build upon this work, for commercial use, provided the original work is properly cited. See: http://creativecommons.org/licenses/ by/4.0/

\section{REFERENCES}

1 Aviña-Zubieta JA, Choi HK, Sadatsafavi M, et al. Risk of cardiovascular mortality in patients with rheumatoid arthritis: a meta-analysis of observational studies. Arthritis Care Res 2008;59:1690-7.
2 Avina-Zubieta JA, Thomas J, Sadatsafavi M, et al. Risk of incident cardiovascular events in patients with rheumatoid arthritis: A meta-analysis of observational studies. Ann Rheum Dis 2012;71:1524-9.

3 Maradit-Kremers $\mathrm{H}$, Crowson CS, Nicola PJ, et al. Increased unrecognized coronary heart disease and sudden deaths in rheumatoid arthritis: A population-based cohort study. Arthritis Rheum 2005;52:402-11.

4 Myasoedova $E$, Chandran A, Ilhan $B$, et al. The role of rheumatoid arthritis (RA) flare and cumulative burden of RA severity in the risk of cardiovascular disease. Ann Rheum Dis 2016;75:560-5.

5 Arts EEA, Fransen J, den Broeder AA, et al. The effect of disease duration and disease activity on the risk of cardiovascular disease in rheumatoid arthritis patients. Ann Rheum Dis 2015;74:998-1003.

6 Greenberg JD, Kremer JM, Curtis JR, et al. Tumour necrosis factor antagonist use and associated risk reduction of cardiovascular events among patients with rheumatoid arthritis. Ann Rheum Dis 2011;70:576-82.

7 Dixon WG, Watson KD, Lunt $M$, et al. Reduction in the incidence of myocardial infarction in patients with rheumatoid arthritis who respond to anti-tumor necrosis factor alpha therapy: results from the British Society for Rheumatology Biologics Register. Arthritis Rheum 2007:56:2905-12.

8 Ljung L, Simard JF, Jacobsson L, et al. Treatment with tumour necrosis factor-inhibitors and the risk of acute coronary syndromes in early rheumatoid arthritis. Arthritis Rheum 2012;64:42.

9 Solomon DH, Avorn J, Katz JN, et al. Immunosuppressive medications and hospitalization for cardiovascular events in patients with rheumatoid arthritis Arthritis Rheum 2006;54:3790-8.

10 Solomon DH, Curtis JR, Saag KG, et al. Cardiovascular risk in rheumatoid arthritis: comparing TNF- $\alpha$ blockade with nonbiologic DMARDs. Am J Med 2013;126:730. e9-e17.

11 Suissa S, Bernatsky S, Hudson M. Antirheumatic drug use and the risk of acute myocardial infarction. Arthritis Care Res 2006;55:531-6.

12 Wolfe F, Michaud K. The risk of myocardial infarction and pharmacologic and nonpharmacologic myocardial infarction predictors in rheumatoid arthritis: A cohort and nested case-control analysis. Arthritis Rheum 2008;58:2612-21.

13 Bili A, Tang X, Pranesh S, et al. Tumor necrosis factor $\alpha$ inhibitor use and decreased risk for incident coronary events in rheumatoid arthritis. Arthritis Care Res 2014;66:355-63.

14 Desai RJ, Rao JK, Hansen RA, et al. Tumor necrosis factor- $\alpha$ inhibitor treatment and the risk of incident cardiovascular events in patients with early rheumatoid arthritis: a nested case-control study. J Rheumatol 2014:41:2129-36.

15 Roubille $C$, Richer $V$, Starnino $T$, et al. The effects of tumour necrosis factor inhibitors, methotrexate, non-steroidal anti-inflammatory drugs and corticosteroids on cardiovascular events in rheumatoid arthritis, psoriasis and psoriatic arthritis: a systematic review and meta-analysis. Ann Rheum Dis 2015;74:480-9.

16 Dixon WG, Symmons DP. What effects might anti-TNFalpha treatment be expected to have on cardiovascular morbidity and mortality in rheumatoid arthritis? A review of the role of TNFalpha in cardiovascular pathophysiology. Ann Rheum Dis 2007;66:1132-6.

17 Kurrelmeyer KM, Michael LH, Baumgarten G, et al. Endogenous tumor necrosis factor protects the adult cardiac myocyte against ischemic-induced apoptosis in a murine model of acute myocardial infarction. Proc Natl Acad Sci USA 2000;97:5456-61.

18 Kleinbongard P, Heusch G, Schulz R. TNFalpha in atherosclerosis, myocardial ischemia/reperfusion and heart failure. Pharmacol Ther 2010:127:295-314.

19 Hyrich KL, Watson KD, Isenberg DA, et al. The British Society for Rheumatology Biologics Register: 6 years on. Rheumatology (Oxford) 2008:47:1441-3.

20 National Institute for Health and Clinical Excellence. Adalimumab, etanercept and infliximab for the treatment of rheumatoid arthritis. October 2007. http://egap. evidence.nhs.uk/TA130

21 Herrett E, Smeeth L, Walker L, et al. The Myocardial Ischaemia National Audit Project (MINAP). Heart 2010;96:1264-7.

22 Thygesen $\mathrm{K}$, Alpert JS, White HD, et al. Universal definition of myocardial infarction. Circulation 2007;116:2634-53.

23 Hyrich $\mathrm{KL}$, Watson KD, Lunt $\mathrm{M}$, et al. Changes in disease characteristics and response rates among patients in the United Kingdom starting anti-tumour necrosis factor therapy for rheumatoid arthritis between 2001 and 2008. Rheumatology (Oxford) 2011;50:117-23.

24 van Buuren S, Boshuizen HC, Knook DL. Multiple imputation of missing blood pressure covariates in survival analysis. Stat Med 1999;18:681-94.

25 Sterne JAC, White IR, Carlin JB, et al. Multiple imputation for missing data in epidemiological and clinical research: potential and pitfalls. BMJ 2009;338: b2393.

26 Gale CP, Manda SOM, Weston CF, et al. Evaluation of risk scores for risk stratification of acute coronary syndromes in the Myocardial Infarction National Audit Project (MINAP) database. Heart 2009;95:221-7.

27 Simms AD, Reynolds S, Pieper K, et al. Evaluation of the NICE mini-GRACE risk scores for acute myocardial infarction using the Myocardial Ischaemia National Audit Project (MINAP) 2003-2009: National Institute for Cardiovascular Outcomes Research (NICOR). Heart 2013;99:35-40. 


\section{Clinical and epidemiological research}

28 Cattle BA, Baxter PD, Greenwood DC, et al. Multiple imputation for completion of a national clinical audit dataset. Stat Med 2011;30:2736-53.

29 Ljung L, Askling J, Rantapää-Dahlqvist S, et al. The risk of acute coronary syndrome in rheumatoid arthritis in relation to tumour necrosis factor inhibitors and the risk in the general population: a national cohort study. Arthritis Res Ther 2014; $16:$ R127

30 Libby P. Inflammation in atherosclerosis. Nature 2002;420:868-74.
31 Daïen $\mathrm{Cl}$, Duny Y, Barnetche T, et al. Effect of TNF inhibitors on lipid profile in rheumatoid arthritis: A systematic review with meta-analysis. Ann Rheum Dis 2012;71:862-8.

32 Gonzalez-Gay MA, Gonzalez-Juanatey C, Vazquez-Rodriguez TR, et al. Insulin resistance in rheumatoid arthritis: the impact of the anti-TNF-alpha therapy. Ann N Y Acad Sci 2010;1193:153-9.

33 Solomon DH, Reed GW, Kremer JM, et al. Disease activity in rheumatoid arthritis and the risk of cardiovascular events. Ann N Y Acad Sci 2015;67:1449-55. 